Int J Adv Manuf Technol (2010) 50:329-341

DOI 10.1007/s00170-009-2487-8

\title{
Availability Modeling and Analysis of Multi-Product
}

\section{Flexible Transfer Lines Subject to Random Failures}

\author{
K. Dhouib, A. Gharbi, N. Landolsi \\ Automated Production Engineering Department, École de Technologie Supérieure, \\ Production System Design and Control Laboratory, \\ University of Québec, 1100 Notre-Dame Street West, Montréal, Québec, Canada H3C 1 K3
}

\begin{abstract}
This paper addresses the problem of estimating the availability of multi-product, flexible transfer lines. Such lines are composed of several machines in series, and can be used to manufacture a variety of products in a batch production environment. The machines are subject to random operation-dependent failures, and there are no intermediate buffers between adjacent machines. This paper aims to propose an analytical model for assessing the steady-state availability of such lines. A simulation model is also developed to mimic the real dynamic behaviour of flexible transfer lines. Thousands of production line configurations are tested in order to compare analytical results with simulation results. We used statistical tests to prove that the proposed formulae are exact and robust to model the steady-state availability of multi-product, unbuffered, flexible transfer lines subject to random failures.
\end{abstract}

Keywords: Flexible transfer lines, Multi-products, Availability assessment, Operationdependent failures, Simulation modelling. 


\section{Introduction}

Production lines are among the most widely studied classes of manufacturing systems [1]. They were originally developed for a cost-efficient mass-production of standardized products, and are characterized by a product flow pattern, and very high production rates $[2,3]$. Thus, a production line composed of $m$ production stages has a product-flow layout in which products are transformed at each stage and flow in order, from stage 1 to stage m.

Two production line classes are often considered: transfer lines and flow lines, with the main feature distinguishing them being that with flow lines, production stages normally involve manual operations, whereas all manufacturing operations are automated in transfer lines. In this paper, we will focus only on the operational issue of automated transfer lines.

Transfer lines are characterized by deterministic operation times and synchronized part transfers from one stage to the next. They can be classified according to workstation failure type and whether or not intermediate buffers are integrated between stages [4]. Buffers are often included to address productivity shortfalls due to equipment failures. Nevertheless, the trends toward lean manufacturing imply a need to reduce and more to eliminate buffers. When modeling transfer lines, two types of failures are often considered: time-dependent failures and operation-dependent failures. However, it must be noted that unlike time-dependent failures, operation-dependent failures cannot occur 
while a workstation is not in operation [5-7]. From a practical point of view, operationdependent failures are more suited to model transfer lines since the age of manufacturing machines depends on their operating horizons [8].

Most of the research that has been carried out to date has dealt with homogeneous transfer lines manufacturing only a single product type $[4,9,10]$. This homogeneous character assumes that transfer lines are perfectly balanced, and so all machine processing times are equal. Product requirements - and thus the requirements of production systems - have changed dramatically from what they were way back to the days of Henry Ford and the famous Model T, and today, ever-changing and more refined customer needs are forcing companies to implement more flexible transfer lines, which has in turn led to mutation towards multi-model or multi-product transfer lines.

This paper studies multi-product, unbuffered, flexible transfer lines subject to random failures. Its aim is to develop an analytical model for assessing the steady-state availability of flexible transfer lines, often also known as line efficiency or effectiveness [11]. The next section reviews the recent literature dealing with mono-product transfer lines. Some reviewed techniques will be extended to the multi-product case, and will be compared to the proposed approach. Section three describes the characteristics of the studied flexible transfer line, and gives notations and assumptions used in this work. Section four proposes a new analytical model for flexible transfer line availability. A simulation model is developed in section five, which is used to validate the precision and the robustness of the proposed analytical model. Numerical results are presented and 
discussed in section six. Finally, in section seven we present concluding remarks and recommendations for future research.

\section{Availability of mono-product transfer lines}

Availability is one of the primary performance measures of transfer lines subject to random failure. Availability in fact affects many other important performance measures, such as transfer line throughput, workstation utilisation rate, and work in process $\underline{[12,13]}$. In this section, we present the primary models used to assess the steady-state availability of mono-product, unbuffered transfer lines.

\subsection{Homogeneous transfer lines}

Homogeneous transfer lines are subject to station interference, i.e., blocking and starvation [14]. If a station completes an operation with the downstream station being failed, it cannot then start a new operation, and it is said to be blocked. Similarly, if a station completes an operation with the upstream station being failed, then it can also not start with a new part, and is said to be starved. From a reliability point of view, homogeneous, unbuffered transfer lines have a serial structure because the entire line stops producing if any one of its machines breaks down. Two formulas are proposed for assessing the steady-state availability (UTR) of homogeneous transfer lines, depending on their failure modes. The UTR of an m-machine, mono-product, unbuffered, homogeneous, transfer line subject to time-dependent failures is given by [4,15-17]: 


$$
U T R=\prod_{i=1}^{m}\left(\frac{1}{1+\frac{\lambda_{i}}{\mu_{i}}}\right)=\prod_{i=1}^{m}\left(\frac{M T B F_{i}}{M T B F_{i}+M T T R_{i}}\right)
$$

However, if the transfer line is subject to operation-dependent failures, its UTR is given by $[4,8,10,15]$ :

$$
U T R=\frac{1}{1+\sum_{i=1}^{m} \frac{\lambda_{i}}{\mu_{i}}}=\frac{1}{1+\sum_{i=1}^{m} \frac{M T T R_{i}}{M T B F_{i}}}
$$

It must be mentioned that the use of equation (1) underestimates the effective availability of transfer lines [8].

\subsection{Non-homogeneous transfer lines}

With homogeneous lines, blocking and starvation result from machine breakdowns. However, if the transfer line is not perfectly balanced, the blocking and starvation can also be caused by differences in machine operation times. In fact, a machine is blocked (starved) on each manufactured part if its processing time is less than that of the immediate downstream (upstream) machine.

Gershwin was the first to propose a disaggregation technique for dealing with nonhomogeneous transfer lines [18]. It decomposes each machine into two equivalent machines having the same production rate as the fastest machine of the entire production line. The first equivalent machine captures the processing time of the original machine, while the second one represents its unreliable behaviour. 
Dallery et al. propose a homogenization technique which consists in replacing each original machine with only one equivalent machine, with all equivalent machines having the same production rate [19]. As was proposed by Gershwin [18], Dallery et al. assign the fastest machine processing time of the original line to all equivalent machines. The equivalent machine parameters are evaluated by considering that the original machine and its equivalent have the same repair process, and the isolated throughput of the original machine and its equivalent are equal.

Liu and Buzacott also use the equivalent machine concept to homogenize nonhomogeneous transfer lines [20]. The processing time of the equivalent line is the same as that of the fastest machine of the original line. Four approaches have been proposed to determine the failure and repair rates of the equivalent machine. Liu and Buzacott find that the approach which considers that "the equivalent machine has the same second moment of interdeparture times" is the most effective.

Chen and Yuan propose that the line be treated somewhat as one whose machines have the same production rate, which is in fact the smallest production rate among the original machines (the bottleneck) [21]. In this case, no modification was introduced to the failure and repair rates of the original line machines. Thus, the transfer line steady-state availability can be simply evaluated through Equation (2).

Dhouib et al. have developed a simulation model to analyse the effectiveness of the four aforementioned proposals in assessing the steady-state availability of unbuffered, nonhomogeneous transfer lines subject to random operation dependant failures [22]. They 
have shown first that the techniques proposed by Gershwin [18], Dallery et al. [19], Liu and Buzacott [20] give the same estimation of mono-product transfer line steady state availability:

$$
U T R=\frac{1}{1+\sum_{i=1}^{n}\left(\frac{\lambda_{i}}{\mu_{i}} \cdot \frac{t_{i}}{t_{\min }}+\frac{t_{i}-t_{\min }}{t_{\min }}\right)}
$$

The study has also revealed that all proposed approaches underestimate the steady-state availability of mono-product transfer lines. Simulation results show that the proposal by Chen and Yuan [21] is the most effective approach when the transfer line includes more than two workstations.

In this paper, the four aforementioned techniques will be extended to the multi-product case, and will be compared to the proposed approach. The next sections present a bi-phase homogenization approach to deal with multi-product flexible transfer lines subject to operation-dependent failures.

\section{Flexible transfer lines: notations, system description, and assumptions}

\subsection{Notations}

Consider a flexible transfer line composed of $\mathrm{m}$ machines and manufacturing $\mathrm{p}$ different products. In this subsection, we present a summary of the notations used in this paper. 
The subscripts $\mathrm{i}$ and $\mathrm{j}$ will be used to denote, respectively, the machine number and the product number in a p-product m-machine flexible transfer line $(i=1,2, \ldots, \mathrm{m}$, and $j=1,2, \ldots, p)$. Therefore, $M_{i}$ denotes the $i^{\text {th }}$ machine of the transfer line and $P_{j}$ denotes the $\mathrm{j}^{\text {th }}$ product manufactured by the transfer line. Generally:

- $\mathrm{L}_{\mathrm{j}}$ : batch or lot size of product $\mathrm{j}$.

- $\mathrm{t}_{\mathrm{i}}$ : part processing time on a mono-product transfer line machine $\mathrm{M}_{\mathrm{i}}$.

- $\mathrm{t}_{\mathrm{bot}}$ : part processing time on the mono-product transfer line bottleneck machine.

- $\mathrm{t}_{\min }$ : part processing time on the mono-product transfer line fastest machine.

- $\mathrm{t}_{\mathrm{ji}}$ : part processing time of product $\mathrm{P}_{\mathrm{j}}$ on machine $\mathrm{M}_{\mathrm{i}}$.

- $\mathrm{t}_{\mathrm{j} \text { bot }}$ : part processing time of product $\mathrm{P}_{\mathrm{j}}$ on the flexible transfer line bottleneck machine.

- $t_{j \min }$ : part processing time of product $\mathrm{P}_{\mathrm{j}}$ on the flexible transfer line fastest machine.

- $T_{j}$ : total time to process a product $P_{j}$ with batch size $L_{j}$ on the flexible transfer line.

- $\lambda_{i}$ : failure rate of machine $M_{i}$.

- $\mu_{\mathrm{i}}$ : repair rate of machine $\mathrm{M}_{\mathrm{i}}$.

- $\mathrm{MTTF}_{\mathrm{i}}$ : mean time to failure of machine $\mathrm{M}_{\mathrm{i}}\left(\mathrm{MTTF}_{\mathrm{i}}=1 / \lambda_{\mathrm{i}}\right)$.

- $\operatorname{MTTR}_{i}:$ mean time to repair of machine $\mathrm{M}_{\mathrm{i}}\left(\mathrm{MTTR}_{\mathrm{i}}=1 / \mu_{\mathrm{i}}\right)$.

- $\lambda_{j i}{ }^{\mathrm{e}}$ : equivalent failure rate of equivalent machine $\mathrm{M}_{\mathrm{i}}$ when manufacturing product $\mathrm{P}_{\mathrm{j}}$.

- $\lambda_{i}^{e}$ : equivalent failure rate of equivalent machine $M_{i}$ for a mono-product transfer line.

- $\mathrm{UR}_{\mathrm{ji}}$ : utilisation rate of machine $\mathrm{M}_{\mathrm{i}}$ when manufacturing product $\mathrm{P}_{\mathrm{j}}$.

- $\mathrm{CF}_{\mathrm{ji}}$ : failure rate correction factor of equivalent machine $\mathrm{M}_{\mathrm{i}}$ when manufacturing product $\mathrm{P}_{\mathrm{j}}$. 
- $\mathrm{MUR}_{\mathrm{i}}$ : mean utilisation rate of equivalent machine $\mathrm{M}_{\mathrm{i}}$ for equivalent mono-product transfer line.

$-\mathrm{CF}_{\mathrm{i}}$ : failure rate correction factor of equivalent machine $\mathrm{M}_{\mathrm{i}}$ for equivalent monoproduct transfer line.

- UTR : steady-state availability of the entire production line.

- $\mathrm{UTR}_{\mathrm{i}}$ : steady-state availability of machine $\mathrm{M}_{\mathrm{i}}$.

- $\mathrm{UTR}_{\mathrm{Tk}}$ : steady-state availability of the flexible transfer line evaluated by Technique $\mathrm{k}$ (Tk).

- $\mathrm{UTR}_{\mathrm{Tk}_{\mathrm{c}} \mathrm{c}}$ : steady-state availability of the $\mathrm{c}^{\text {th }}$ transfer line configuration based on Technique $\mathrm{k}$.

- $\mathrm{UTR}_{\mathrm{s}}$ : steady-state availability of the flexible transfer line evaluated by simulation model.

- $\mathrm{UTR}_{\mathrm{s}}{ }^{\mathrm{r}}$ : steady-state availability of the flexible transfer line evaluated by simulation model for replication $r$.

- $\mathrm{UTR}_{\mathrm{s}_{-} \mathrm{c}}$ : steady-state availability of the $\mathrm{c}^{\text {th }}$ line configuration evaluated by simulation model.

- UTR_RE : relative error given by comparing analytical availability to simulation availability.

- UTR_MRE : mean relative error given by comparing analytical availability to simulation availability for all configurations of a p-product, m-machine flexible transfer line.

- PPwF : parts produced during simulation horizon when the transfer line is subject to random failures. 
- PPoF : parts produced during simulation horizon when the transfer line is perfectly reliable.

\subsection{System description}

The flexible transfer line studied in this paper is an automated production line having multiple machines set out in a serial structure, and interconnected through an automatic transfer mechanism. The flexible transfer line allows the manufacture of several products in a batch production environment. No intermediate buffers are included between machines. Each product type flows from outside the transfer line to the first machine $\left(\mathrm{M}_{1}\right)$, and then passes through all machines until it reaches the last machine $\left(\mathrm{M}_{\mathrm{m}}\right)$, after which it leaves the transfer line.

The flexible transfer line is dedicated to producing $\mathrm{p}$ products in batches with known and finite sizes. Each product $P_{j}$ is manufactured in a batch of $L_{j}$ parts. To be processed, a part requires a deterministic amount of time $\left(t_{\mathrm{ji}}\right)$ depending on the product type $\left(\mathrm{P}_{\mathrm{j}}\right)$ and the

machine $\left(\mathrm{M}_{\mathrm{i}}\right)$ being used to produce it. Because of a lack of balancing multi-product transfer lines, the processing times from one machine to another are different, and the flexible transfer line is non-homogeneous. Figure 1 shows a p-product m-machine flexible transfer line.

The flexible transfer line manufactures the products in a cyclic manner (Fig. 2): $\mathrm{P}_{1}, \mathrm{P}_{2}$, $\ldots, \mathrm{P}_{\mathrm{p}}, \mathrm{P}_{1}, \mathrm{P}_{2}, \ldots, \mathrm{P}_{\mathrm{p}}, \ldots$ Furthermore, every product flows from machine 1 to machine $\mathrm{m}$ in order, and requires all machines to be considered as finished product. 
The flexible transfer line machines are subject to random failures. When a failure occurs, the upstream machine is blocked, and the downstream one starved, until the machine which failed is repaired. Blockage and starvation also occur as a result of the nonhomogeneous character of the flexible transfer line.

\subsection{Working assumptions}

A1- The line operates under saturation: the first station is never starved due to lack of raw parts and the last one is never blocked due to lack of space.

A2- Failures are operation-dependent [8].

A3- Failure times and repair times are exponentially distributed random variables.

A4- On failure, parts remain on machines and processing resumes after repair completion.

A5- Transfer times between machines are considered negligible.

A6- No parts are scrapped.

\section{Availability modeling of multi-product flexible transfer lines}

Most of the research conducted until now has assumed that transfer lines are dedicated to producing a single product type. Savsar and Biles mention that the manufactured product can be assumed to be an aggregate of multiple product types [23]. However, no author has indicated how to switch from a mono-product to a multi-product configuration. 
Indeed, modeling and analysing flexible transfer lines is a more complex endeavour since they imply: a) the existence of a non-homogeneous operational set-up, and b) various product types with additional parameters (specific product manufacturing times and product lot sizing).

This section proposes a new model for assessing the steady-state availability of multiproduct, unbuffered, flexible transfer lines subject to random failures. It is based on a homogenization approach which converts the discrete intermittent operating behaviour of the flexible transfer line into an equivalent one with a continuous operating behaviour.

Consider the effective discrete workload of a flexible transfer line machine $\mathrm{M}_{\mathrm{i}}$ (Fig. 3). We note that machine $M_{i}$ operates intermittently when manufacturing products $P_{1}$ and $P_{3}$, for example. However, it operates continuously when manufacturing product $\mathrm{P}_{2}$. This behaviour depends on the position of the bottleneck machine with respect to the manufactured product; machine $\mathrm{M}_{\mathrm{i}}$ is the bottleneck machine when manufacturing product $\mathrm{P}_{2}$, but not so when manufacturing products $\mathrm{P}_{1}$ and $\mathrm{P}_{3}$. Since the machine's failure mode is operation-dependent, it means machine $M_{i}$ does not age during idle periods. Hence, for a specific product $\mathrm{P}_{\mathrm{j}}$, and for each part produced, the period during which a machine $M_{i}$ is idle is equal to $t_{j \text { bot }}-t_{j i}$.

Based on these observations, the proposed homogenization approach consists of two phases: homogenization by product type and global homogenization. 


\subsection{Homogenization by product type}

The first step of the homogenization approach consists in isolating each manufactured product and converting the discrete operating behaviour of each original machine into a continuous mode for each manufactured product (Fig. 4).

Consequently, each of the equivalent flexible transfer line machines is assumed to be operating in a continuous manner when manufacturing each product type with a reduced capacity and the reduced capacity depends on the machine utilisation rate for each machine-product pair.

Accordingly, the original flexible transfer line can be assimilated into an equivalent one with machines operating continuously. For each specific product type $\mathrm{P}_{\mathrm{j}}$, every equivalent transfer line machine $\mathrm{M}_{\mathrm{i}}$ has a processing time equal to that of the bottleneck machine

$\left(t_{j b o t}\right)$, an equivalent failure rate $\left(\lambda_{j i}{ }^{e}\right)$, and a repair rate equal to that of the corresponding original machine $\left(\mu_{\mathrm{i}}\right)$.

The equivalent failure rate of the equivalent machine when manufacturing product $\mathrm{P}_{\mathrm{j}}$ is equal to the failure rate of the original machine when operating at full capacity multiplied by a correction factor $\mathrm{CF}_{\mathrm{ji}}$ :

$$
\lambda_{j i}^{e}=\lambda_{i} \cdot C F_{j i}
$$


The failure rate correction factor $\mathrm{CF}_{\mathrm{ji}}$ is equal to the utilisation rate of the original machine $\mathrm{M}_{\mathrm{i}}$ when manufacturing product $\mathrm{P}_{\mathrm{j}}$ :

$$
C F_{j i}=U R_{j i}=\frac{t_{j i}}{t_{j b o t}}
$$

where: $t_{j b o t}=\max _{i=1}^{m}\left(t_{j i}\right)$

\subsection{Global homogenization}

The second step of the homogenization approach consists in converting the workload of an equivalent flexible transfer line machine $\mathrm{M}_{\mathrm{i}}$ (Fig. 4), into a steady and continuous workload (Fig. 5).

Hence, the original multi-product flexible transfer line is assimilated into a homogeneous equivalent one manufacturing just a single product type, and operating continuously, with steady capacity. Each equivalent mono-product transfer line machine $M_{i}$ has an equivalent failure rate $\left(\lambda_{i}{ }^{e}\right)$, and a repair rate equal to that of the corresponding original multi-product machine $\left(\mu_{\mathrm{i}}\right)$. The equivalent failure rate of any equivalent mono-product transfer line machine which has been estimated by the weighted average of all equivalent failure rates of the equivalent multi-product machine $\mathrm{M}_{\mathrm{i}}$ (Eq. 4); it can be obtained by:

$$
\lambda_{i}^{e}=\frac{\sum_{j=1}^{p} \lambda_{j i}^{e} \cdot T_{j}}{\sum_{j=1}^{p} T_{j}}
$$


The weight corresponding to a specific value $\lambda_{\mathrm{ji}}{ }^{\mathrm{e}}$ can be estimated by the total time required to manufacture the entire batch of the product $P_{j}$ on the flexible transfer line $\left(T_{j}\right)$, where $T_{j}$ is given by:

$$
T_{j}=t_{j b o t} \cdot L_{j}
$$

By replacing $\lambda_{\mathrm{ji}}{ }^{\mathrm{e}}$ and $\mathrm{CF}_{\mathrm{ji}}$ with their corresponding values (Eqs. 4, 5), the equivalent failure rate of any equivalent mono-product transfer line machine $\mathrm{M}_{\mathrm{i}}$ can be simplified as follows:

$$
\lambda_{i}^{e}=\lambda_{i} \cdot \frac{\sum_{j=1}^{p} t_{j i} \cdot L_{j}}{\sum_{j=1}^{p} t_{j b o t} \cdot L_{j}}
$$

Thus, the equivalent failure rate of any equivalent mono-product transfer line machine is equal to the failure rate of the original machine $M_{i}$ when operating at full capacity, multiplied by a correction factor denoted as $\mathrm{CF}_{\mathrm{i}}$ :

$$
C F_{i}=M U R_{i}=\frac{\sum_{j=1}^{p} t_{j i} \cdot L_{j}}{\sum_{j=1}^{p} t_{j b o t} \cdot L_{j}}
$$

The failure rate correction factor $\mathrm{CF}_{\mathrm{i}}$ is estimated through the mean utilisation rate of machine $\mathrm{M}_{\mathrm{i}}$ when manufacturing all product types. Therefore, the steady-state availability of a multi-product, unbuffered, flexible transfer line subject to operationdependent failures can be evaluated using equation (2), and replacing $\lambda_{\mathrm{i}}$ with $\lambda_{\mathrm{i}}{ }^{\mathrm{e}}$ (Eq. 8). 
In the case where all manufacturing products have the same batch size, the equivalent failure rate of a mono-product equivalent machine $\mathrm{M}_{\mathrm{i}}$ is reduced to:

$$
\lambda_{i}^{e}=\lambda_{i} \cdot \frac{\sum_{j=1}^{p} t_{j i}}{\sum_{j=1}^{p} t_{j b o t}}
$$

Furthermore, if the flexible transfer line manufactures only one product type $(\mathrm{p}=1)$, the equivalent failure rate of an equivalent machine $\mathrm{M}_{\mathrm{i}}$ can be obtained by:

$$
\lambda_{i}^{e}=\lambda_{i} \cdot \frac{t_{i}}{t_{b o t}}
$$

where $t_{i} / t_{b o t}$ is the utilisation rate of a mono-product non-homogeneous transfer line machine $\mathrm{M}_{\mathrm{i}}$ when operating without failures.

\section{Multi-product flexible transfer line simulation model}

To assess the effective value of the steady-state availability of multi-product, unbuffered, flexible transfer lines subject to operation-dependent failures, a general discrete event simulation model was developed with the AweSim/VisualSlam system [24]. The simulation model allows the evaluation of the performance of the proposed bi-phase homogenization approach and its associated model in assessing flexible transfer line availability. It also analyses the performance of extended versions of equivalent techniques by Gershwin [18], Dallery and al. [19], Liu and Buzacott [20], and the proposal by Chen and Yuan's [21] to the multi-product case. The simulation model 
mimics the actual dynamic and stochastic behaviours of flexible transfer lines. It can be used with various numbers of machines, different product types, lot sizes, processing times, and failure and repair rates. The flow chart of the simulation model is given in Figure 6, with the following description of its four principal modules:

1- INITIALIZATION: sets, for each experiment, the number of line machines, the number of product types, the lot sizes, the processing times, the mean time to failure, and the mean time to repair for each machine. The simulation horizon and the warmup period after which statistics are cleared are also assigned at this step.

2- PRODUCTION: controls the flow of parts through the production line. Blocking and starvation due to failures and to the non-homogeneous character of production lines are managed in this module.

3- FAILURE and REPAIR: sample times to failure and repair durations for each machine from their respective probability distributions. This module allows changes in machine state and adjusts, for each machine, the effective failure instance according to the operation-dependent failure mode.

4- UPDATE PERFORMANCE: saves the number of parts produced during simulation horizon. This allows an assessment of the production line steady-state availability:

$$
U T R_{s}=\frac{P P w F}{P P o F}
$$

where PPoF can be evaluated using the following equation: 


$$
\text { PPoF }=\frac{\text { Simulation Horizon }}{\sum_{j=1}^{p} t_{j b o t} \cdot L_{j}} \cdot \sum_{j=1}^{p} L_{j}
$$

The simulation ends when the current simulation time TNOW reaches the simulation horizon.

The effective availability given by the simulator is compared to that given by each studied technique through the Student's t-test, with the following $\mathrm{H}_{0}$ hypothesis:

$\mathrm{H}_{0}$ : the availability given by the analytical formula $\left(\mathrm{UTR}_{\mathrm{TK}}\right)$ is not different from the effective one generated by the simulation model $\left(\mathrm{UTR}_{\mathrm{s}}\right)$.

For each line configuration, $\mathrm{N}$ simulation replications are performed to obtain $\mathrm{N}$ incurred $\mathrm{UTR}_{\mathrm{s}}$ observations. For the 1- $\alpha$ confidence level, $\mathrm{H}_{0}$ is accepted if the value of the t-distribution with $v$ degrees of freedom and a confidence level 1- $\alpha$ (i.e., $t_{v, 1-\alpha / 2}$ for two sided tests) is greater than the absolute value of $\mathrm{t}_{0}$ given by:

$$
t_{0}=\frac{\overline{U T R_{s}}-U T R_{T k}}{\sqrt{S^{2} / N}}
$$

where: $\quad v=N-1, \quad \overline{U T R_{s}}=(1 / N) \cdot \sum_{r=1}^{N} U T R_{s}^{r}, \quad S^{2}=(1 /(N-1)) \cdot \sum_{r=1}^{N}\left(U T R_{s}^{r}-\overline{U T R_{s}}\right)^{2}$

Each simulation is executed during a horizon whose length depends on the number of products manufactured by the flexible transfer line. A 100,000 unit time warm-up period 
has been considered to guarantee the stability of performance measures. 10 replications were realised with each production line configuration. So, For $\mathrm{N}=10$ and $\alpha=5 \%$, the Student's t-test table gives $t_{9,0.975}=2.262$. Therefore, the Hypothesis $\mathrm{H}_{0}$ is accepted if $\left|t_{0}\right| \leq 2.262$.

\section{Numerical examples}

Consider a multi-product flexible transfer line composed of $\mathrm{m}$ stations, and manufacturing $\mathrm{p}$ products. In this study we have analysed thousands of flexible transfer lines with a number of stations $m$ varying between 3 and 10 , and a number of products $p$ being equal to 3,5 , and 9 products.

To analyse the effect of individual machine availability on global production line availability, two ranges for individual machine availability are considered: low availability $\left(\mathrm{UTR}_{\mathrm{i}} \in[70,75 \%]\right)$, and high availability $\left(\mathrm{UTR}_{\mathrm{i}} \in[95,100 \%]\right)$. The input data utilised for these configurations are given below.

\subsection{Input data}

In this research, and for each configuration of the multi-product flexible transfer line, all parameters were generated randomly by following the next procedure:

A- For each product $P_{j}$ manufactured by the flexible transfer line $(j=1,2, \ldots, p)$ :

1- Select randomly a machine to be a bottleneck one ( $b o t=1,2, \ldots$ or $\mathrm{m})$ 
2- Randomly generate the processing time on the bottleneck: $t_{j}$ bot $\in[10,25]$ (time units).

3- For each machine $\mathrm{M}_{\mathrm{i}}$ of the $\mathrm{m}$-machine flexible transfer line $(\mathrm{i}=1,2, \ldots, \mathrm{m})$ :

a. Randomly generate the utilisation rate of machine $\mathrm{M}_{\mathrm{i}}: \mathrm{UR}_{\mathrm{ji}} \in[70 \%, 100 \%]$ and $\mathrm{i} \neq \mathrm{bot}$

b. Evaluate, for $\mathrm{i} \neq$ bot, the machine processing time by:

$$
t_{j i}=U R_{j i} \cdot t_{j b o t}
$$

4- Randomly generate the lot size of product $\mathrm{P}_{\mathrm{j}}$ : $\mathrm{L}_{\mathrm{j}} \in[500,1000]$ (parts).

B- For each machine $\mathrm{M}_{\mathrm{i}}$ of the flexible transfer line $(\mathrm{i}=1,2, \ldots, \mathrm{m})$ :

5- Randomly generate the individual availability of machine $\mathrm{M}_{\mathrm{i}}\left(\mathrm{UTR}_{\mathrm{i}}\right)$.

6- Randomly generate its mean time to repair: $\operatorname{MTTR}_{\mathrm{i}} \in[30,100]$ (time units).

7- Calculate its mean time to failure by the following equation:

$$
M T T F_{i}=M T T R_{i} \cdot\left(\frac{U T R_{i}}{1-U T R_{i}}\right)
$$

A Visual Basic program was developed to automatically generate, and according to the above procedure, thousands of random configurations of flexible transfer lines.

\subsection{Numerical Results}

To analyse the performance of the studied models, many programs were developed using Visual Basic programming language. These programs contributed to automating several 
processes used in generating and analysing results: random configuration generation, evaluation of analytical models, analysis of simulation results, and evaluation of Student's t-tests and errors generated by comparing analytical results with effective simulation results (Fig. 7).

Table 1 gives, for example, ten randomly generated configurations for a flexible transfer line having three workstations and manufacturing three products. It gives the characteristics of manufactured products and transfer line workstations. Based on the proposed analysis methodology (Fig. 7) and experiments of Table 1, Table 2 consequently summarizes the steady-state availability generated by the simulation model, by the proposed technique (DGL), by the proposal by Chen and Yuan (C\&Y) [21], and by the equivalent technique (GDLB) proposed by Gershwin [18], Dallery et al. [19], and Liu and Buzacott [20]. For the equivalent GDLB technique, the flexible transfer line steady state availability can be evaluated by:

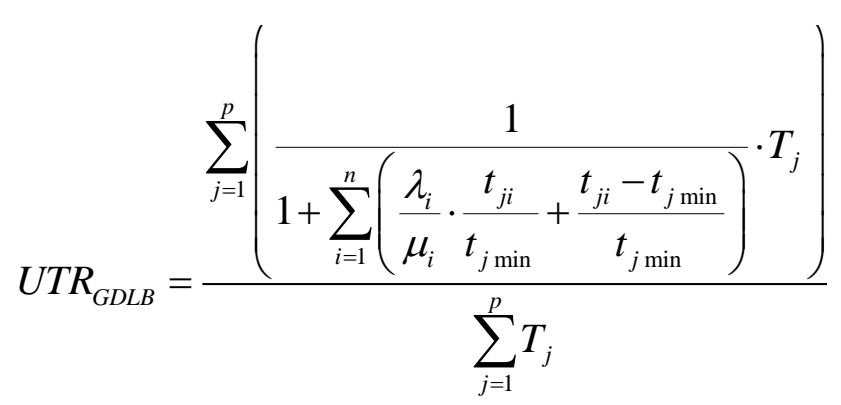

Table 2 also states the relative availability errors (Eq. 18), and the Student's t-test acceptance by comparing analytical results with those obtained through simulation. 


$$
\mathrm{UTR} \_\mathrm{RE}(\%)=\frac{\overline{U T R}_{s_{-} c}-U T R_{T k_{-} c}}{\overline{U T R}_{s_{-} c}} \cdot 100 \%
$$

where: $\mathrm{c}$ : is the randomly generated $\mathrm{c}^{\text {th }}$ configuration of a p-product, m-machine transfer line.

$\overline{U T R}_{s_{-} c}$ : is the mean simulated UTR of the $c^{\text {th }}$ line configuration (mean of 10 replications).

Tk : technique to evaluate flexible transfer line availability (Tk = DGL, GDLB, C\&Y).

Tables 3 and 4 show the values of UTR mean relative errors (Eq. 19) - mean for 100 randomly generated configurations for each p-product, m-machine flexible transfer line and the percentage of successful Student's t-test obtained by comparing results of each technique with simulation results, respectively when $\mathrm{UTR}_{\mathrm{i}} \in[70,75 \%]$ and $\mathrm{UTR}_{\mathrm{i}} \in[95,100 \%]$.

$$
\operatorname{UTR\_ MRE~}(\%)=\frac{\left|\sum_{c=1}^{100} \frac{\overline{U T R}_{s_{-} c}-U T R_{T k_{-} c}}{\overline{U T R}_{s_{-} c}}\right|}{100} \cdot 100 \%
$$

\subsection{Discussion}

The results given in Tables 3 and 4 show first that the proposed technique (DGL) generates a negligible error compared with simulation results for all flexible transfer line configurations. The mean relative error remains under $0.2 \%$ for all analysed configurations, irrespective of transfer line length, the number of products being 
manufactured, and the individual machine availability range. The results also show that hypothesis $\mathrm{H}_{0}$ is accepted for all line configurations when using the proposed technique. This confirms that the steady-state availability formulations for multi-product, unbuffered flexible transfer lines based on the proposed technique are accurate and robust.

However, Tables 3 and 4 show that the relative error generated by the extended techniques to the multi-product flexible transfer lines by Gershwin [18], Dallery et al. [19], Liu and Buzacott [20], and Chen and Yuan [21] increases accordingly with the number of the flexible transfer line machines. Furthermore, and compared to simulation results, all these techniques underestimate effective availability of flexible transfer lines.

In the other hand, figure 8 explains that the error generated by techniques GDLB and C\&Y does not fluctuate significantly when the number of product types being manufactured by the flexible transfer line increases. Finally, and as observed by Dhouib et al. in the case of mono-product transfer lines [22], technique C\&Y outperforms equivalent technique GDLB. Effectively, C\&Y technique generates less error than GDLB technique.

\section{Conclusions and recommendations}

This paper examined the assessment of the steady-state availability of multi-product, unbuffered flexible transfer lines subject to random operation-dependent failures. A bi-phase homogenization approach was proposed, which consists in transforming the 
discrete intermittent operating behaviour of the flexible transfer line into a steady and continuous operating mode. Based on this homogenization approach, analytical formulations are also proposed to evaluate the steady-state availability of flexible transfer lines.

In order to analyse the precision and robustness of the proposed homogenization approach and its resulting analytical formulations, a general discrete event simulation model was developed. It allows the mimicking of the actual dynamic and stochastic behaviours of such flexible transfer lines. Thousands of production line configurations with different lengths, product types, and randomly generated machine processing times, lot sizes, failure and repair rates were tested (lines including from 3 to 10 machines and manufacturing 3,5 , or 9 products).

Comparing analytical and simulation results, we can show that extended techniques to the multi-product case by Gershwin [18], Dallery et al. [19], Liu and Buzacott [20], and Chen and Yuan [21] lead to significant errors, and underestimate the effective availability of flexible transfer lines. On the other hand, the proposed homogenization technique suited the simulation results for all randomly generated transfer line configurations perfectly. Furthermore, statistical tests confirm the precision and robustness of the proposed formulae.

This research should be extended to develop analytical models for analysing more complicated systems, such as multi-stage production lines with inventory banks, assembly/disassembly systems, and cellular production systems. 


\section{References}

[1] Buzacott JA, Shanthikumar JG. Design of Manufacturing Systems Using Queueing models. Queueing Systems 1992; 12:135-213.

[2] Shtub A, Dar-El EM. A Methodology of the Selection of Assembly Systems. Int J Prod Res $1989 ; 27: 175-186$.

[3] Scholl A. Balancing and Sequencing Assembly Lines. New-York: Heidelberg Physica; 1999.

[4] Papadopoulos HT, Heavey C. Queueing theory in manufacturing systems analysis and design: A classification of models for production and transfer lines. Eur J Oper Res 1996; 92:1-27.

[5] Sherwin DJ. Steady-State Series Availability. IEEE Trans Reliab 2000; 49:146-147.

[6] Pham H. Commentary: Steady-State Series-System Availability. IEEE Trans Reliab 2003; 52:131-132.

[7] Schneeweiss WG. Toward a Deeper Understanding of the Availability of series-Systems Without Aging During Repairs. IEEE Trans Reliab 2005; 54:98-99.

[8] Buzacott JA. Prediction of the efficiency of production systems without internal storage. Int $\mathbf{J}$ Prod Res 1968; 6:173-188.

[9] Dallery Y, Gershwin SB. Manufacturing Flow line systems: A review of models and analytical results. Queueing Systems 1992; 12:3-94.

[10] Buzacott JA, Shanthikumar JG. Stochastic Models of Manufacturing Systems. New Jersey: Prentice-Hall; 1993.

[11] Askin RG, Standridge CR. Modeling and Analysis of Manufacturing Systems. New-York: John Wiley \& Sons; 1993.

[12] Freiheit T, Koren Y, Hu J. Productivity of Parallel Production Lines with Unreliable Machines and Material Handling. IEEE Trans Aut Sci Eng 2004; 1:98-103.

[13] Limnios N. Throughput Availability in Markov Systems. IEEE Trans Reliab 1992; 41:219-224. 
[14] Yeralan S, Muth EJ. A general model of a production line with intermediate buffers and station breakdowns. IIE Trans 1987; 19:130-139.

[15] Gershwin SB. Manufacturing Systems Engineering. New-York: Prentice-Hall; 1994.

[16] Tan B. Variance of the throughput of an N-station production line with no intermediate buffers and time-dependent failures. Eur J Oper Res 1997; 101:560-576.

[17] Der Kiureghian A, Ditlevsen OD, Song J. Availability, reliability and downtime of systems with repairable components. Reliab Eng Syst Saf 2007; 92: 231-242.

[18] Gershwin SB. Representation and analysis of transfer lines with machines that have different processing rates. Annals Oper Res 1987; 9:511-530.

[19] Dallery Y, David R, Xie XL. Approximate Analysis of Transfer Lines with Unreliable Machines and Finite Buffers. IEEE Trans Aut Cont 1989; 34:943-953.

[20] Liu XG, Buzacott JA. Approximate models of assembly systems with finite inventory banks. Eur J Oper Res 1990; 45:143-154.

[21] Chen CT, Yuan J. Transient throughput analysis for a series type system of machines in terms of alternating renewal processes. Eur J Oper Res 2004; 155:178-197.

[22] Dhouib K, Gharbi A, Ayed S. Throughput Assessment of Non-Homogeneous Transfer Lines: Simulation Modeling Based Approach. In: Proceedings of the International Conference on Industrial Engineering and Systems Management (IESM’07), Beijing, China, 2007.

[23] Savsar M, Biles WE. Two-Stage Production Lines with a Single Repair Crew. Int J Prod Res 1984; 22:499-514.

[24] Pritsker AA, O’Reilly JJ. Simulation with Visual SLAM and AweSim. New York: John Wiley \& Sons; 1999. 


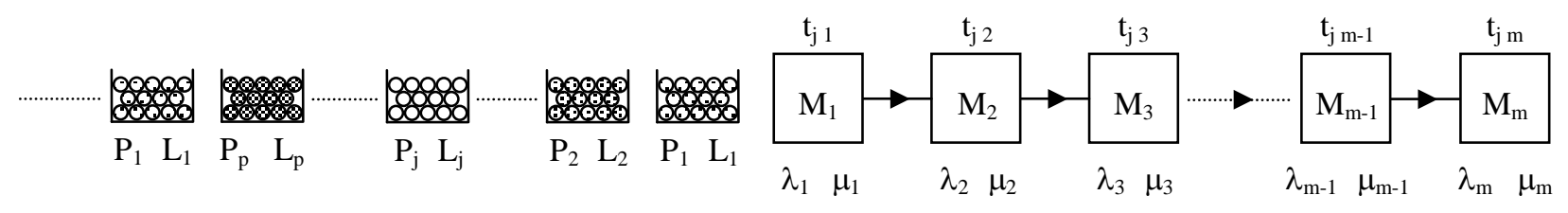

Fig. 1. p-product, m-machine flexible transfer line.

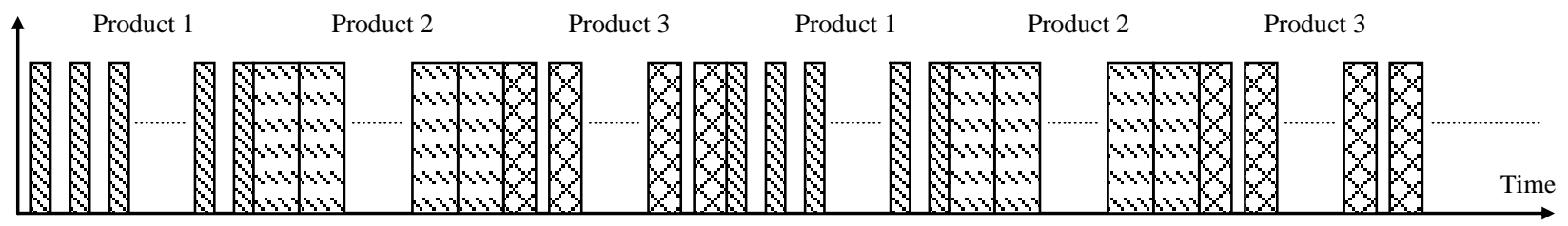

Fig. 2. Workload of a 3-product flexible transfer line machine.

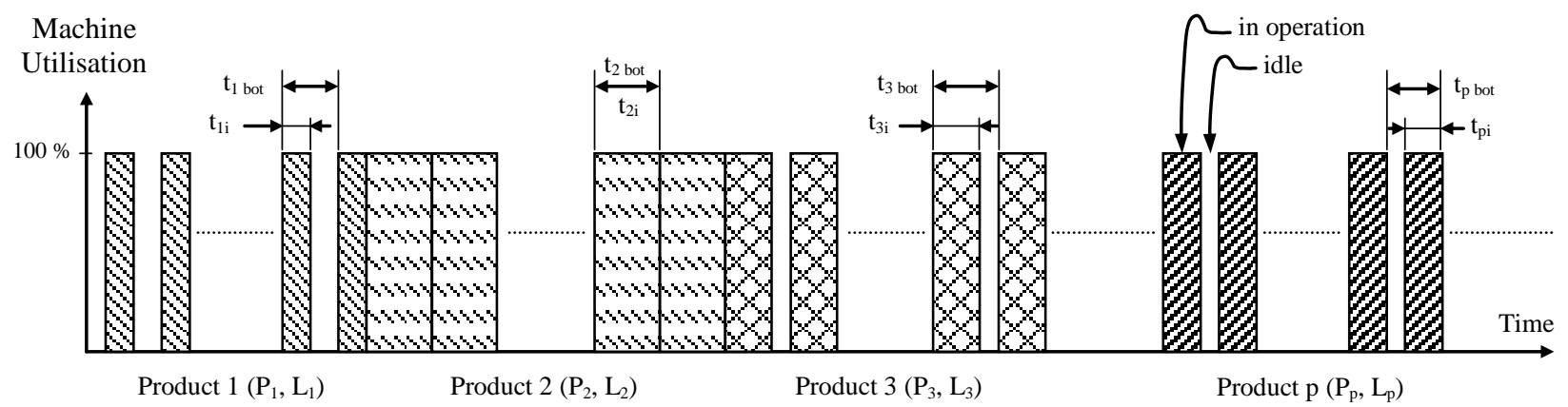

Fig. 3. Workload of a typical p-product machine $\mathrm{M}_{\mathrm{i}}$ (discrete mode).

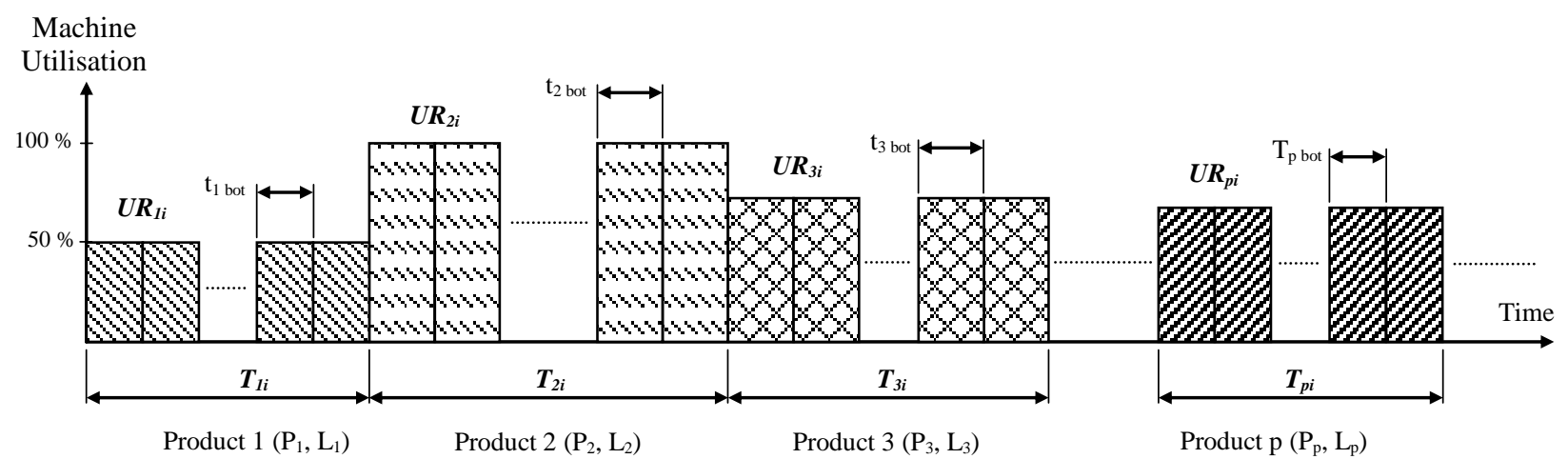

Fig. 4. Workload of equivalent p-product machine $\mathrm{M}_{\mathrm{i}}$ (continuous mode). 


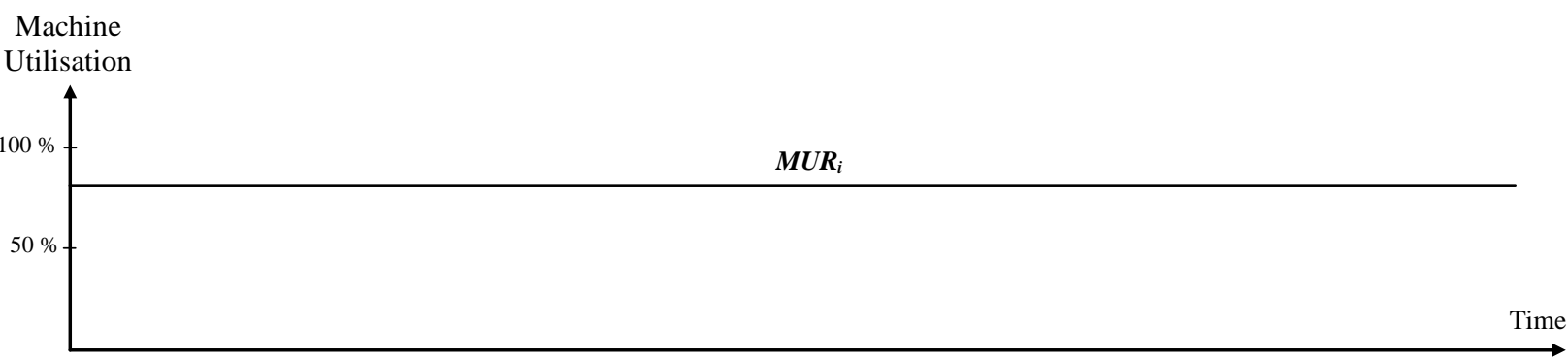

Fig. 5. Workload of equivalent mono-product machine $M_{i}$ (continuous mode).

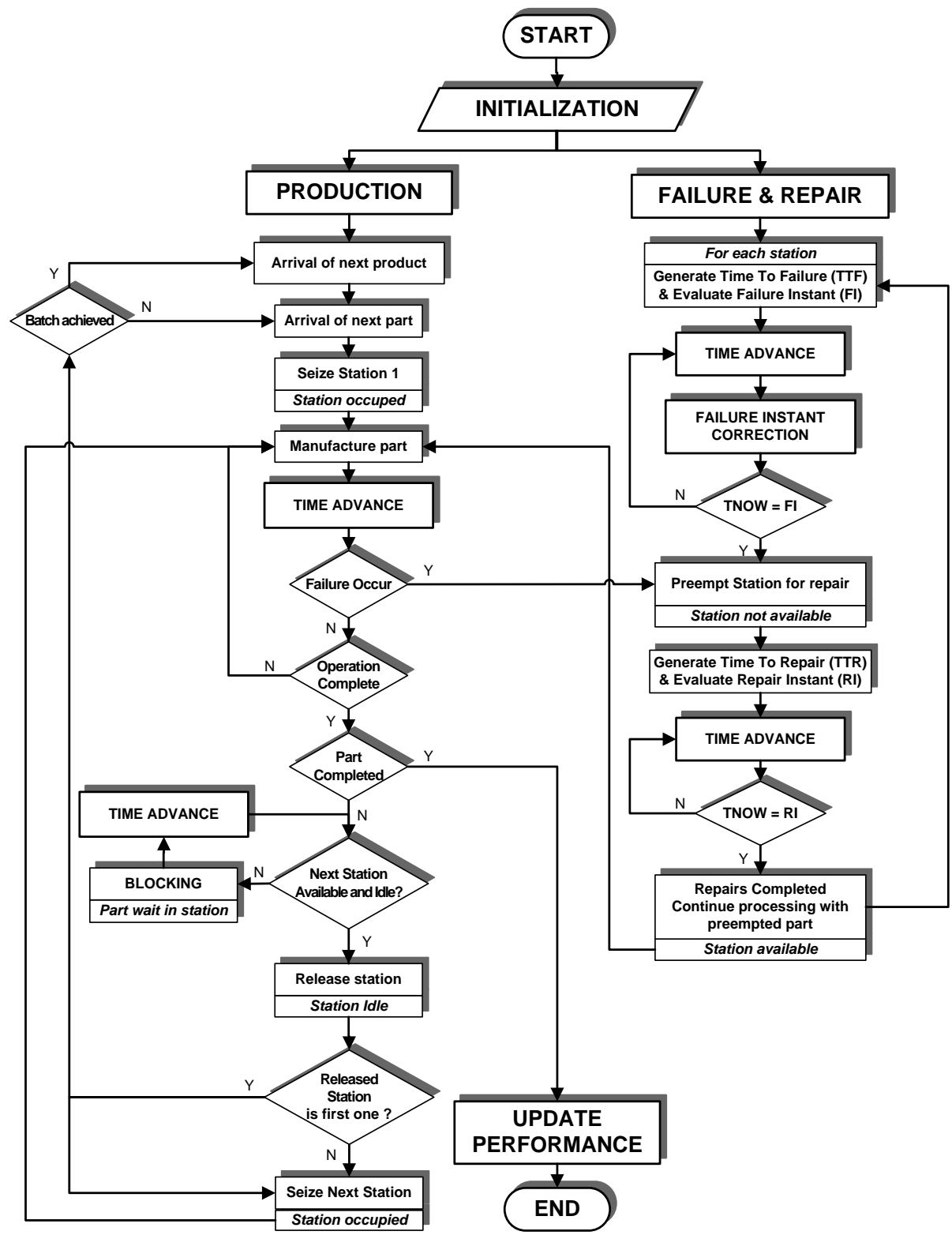

Fig. 6. General discrete event simulation model for multi-product flexible transfer lines. 


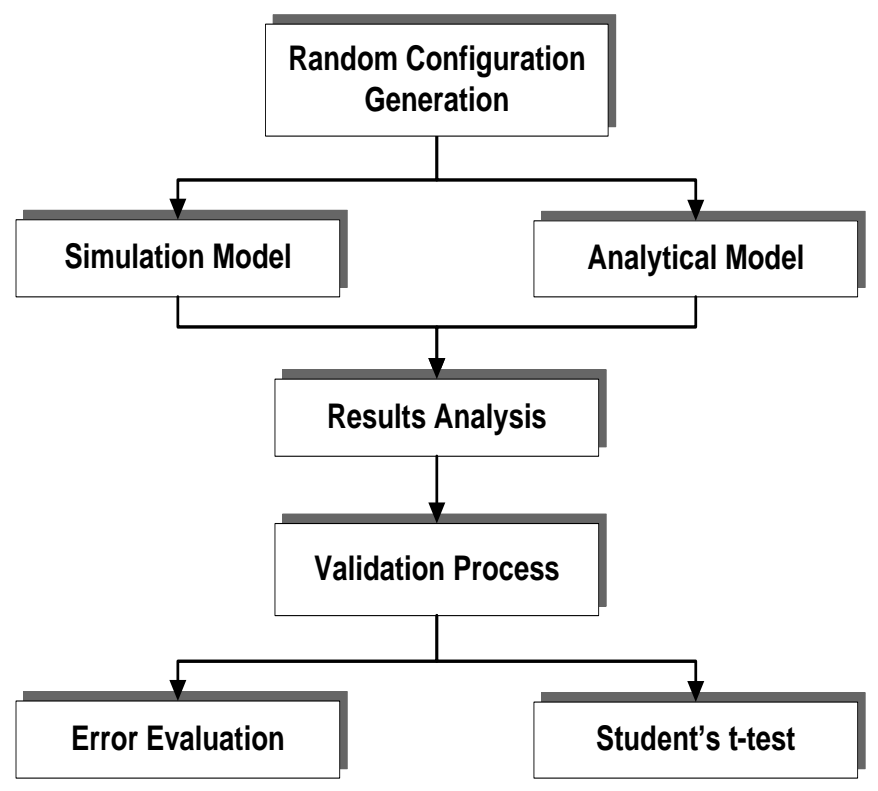

Fig. 7. Analysis methodology for comparing analytical results with simulation results.

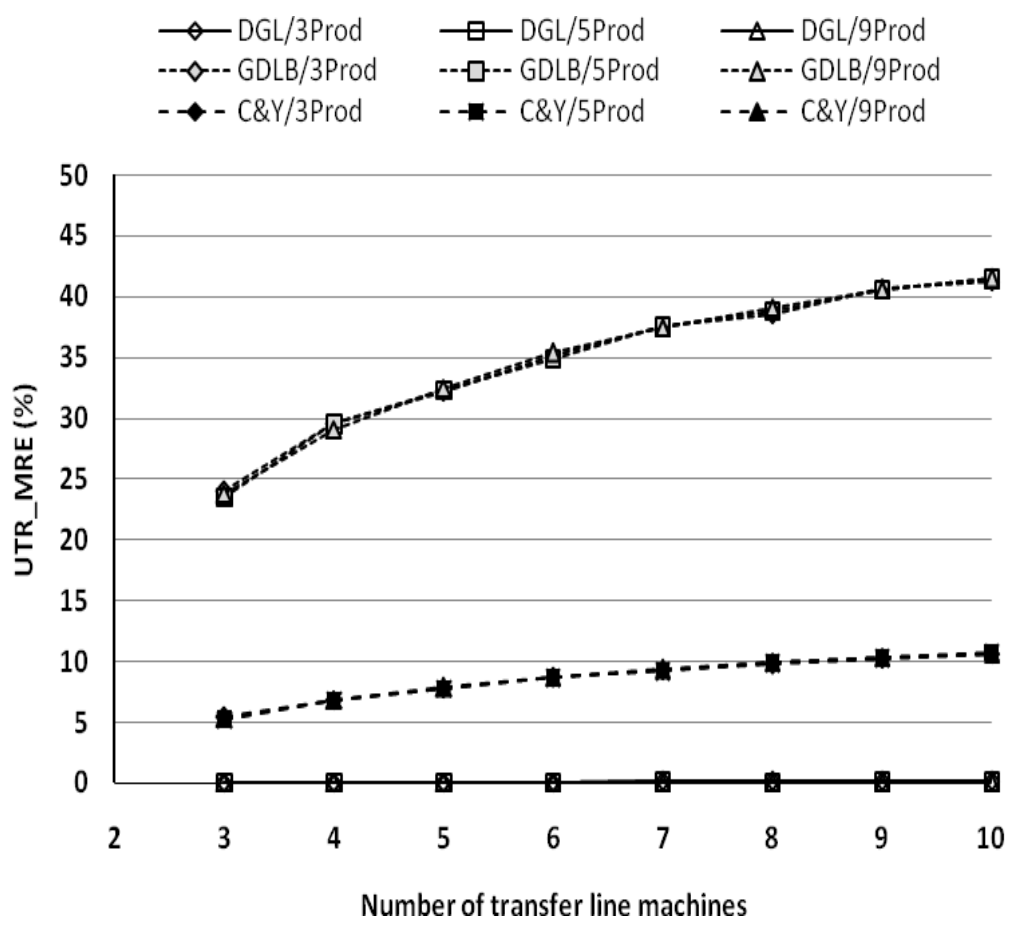

Fig. 8. UTR mean relative error of studied techniques versus simulation $\left(\mathrm{UTR}_{\mathrm{i}} \in[70,75 \%]\right)$. 


\begin{tabular}{|c|c|c|c|c|c|c|c|c|c|c|c|c|}
\hline \multirow[b]{2}{*}{$\begin{array}{l}\text { Flexible transfer } \\
\text { line configurations }\end{array}$} & \multicolumn{4}{|c|}{ Product 1} & \multicolumn{4}{|c|}{ Product 2} & \multicolumn{4}{|c|}{ Product 3} \\
\hline & $\mathrm{t}_{11}$ & $\mathrm{t}_{12}$ & $\mathrm{t}_{13}$ & $\mathrm{~L}_{1}$ & $t_{21}$ & $t_{22}$ & $\mathrm{t}_{23}$ & $\mathrm{~L}_{2}$ & $\mathrm{t}_{31}$ & $t_{32}$ & $t_{33}$ & $\mathrm{~L}_{3}$ \\
\hline 1 & 17.99 & 20.58 & 16.20 & 650 & 21.62 & 20.07 & 20.42 & 854 & 10.24 & 10.68 & 10.01 & 686 \\
\hline 2 & 17.51 & 24.06 & 24.43 & 682 & 12.80 & 15.69 & 17.87 & 734 & 12.94 & 14.47 & 11.28 & 639 \\
\hline 3 & 19.68 & 22.35 & 22.45 & 955 & 13.32 & 10.36 & 13.40 & 766 & 10.47 & 8.17 & 11.60 & 787 \\
\hline 4 & 11.50 & 10.81 & 9.03 & 522 & 11.41 & 14.44 & 14.21 & 989 & 16.02 & 11.99 & 12.00 & 823 \\
\hline 5 & 14.76 & 16.15 & 12.89 & 816 & 13.11 & 11.47 & 9.50 & 728 & 23.59 & 22.07 & 19.19 & 644 \\
\hline 6 & 21.13 & 23.79 & 19.71 & 548 & 17.94 & 17.50 & 18.42 & 511 & 15.05 & 16.40 & 18.15 & 751 \\
\hline 7 & 14.27 & 17.71 & 14.54 & 634 & 10.83 & 10.77 & 7.78 & 695 & 11.56 & 15.47 & 13.04 & 628 \\
\hline 8 & 14.51 & 19.43 & 19.07 & 827 & 12.88 & 17.59 & 16.45 & 729 & 20.24 & 21.31 & 15.03 & 605 \\
\hline 9 & 11.11 & 8.88 & 8.20 & 500 & 15.58 & 18.05 & 17.12 & 540 & 10.77 & 10.39 & 12.88 & 574 \\
\hline 10 & 17.67 & 14.95 & 20.57 & 878 & 13.59 & 16.03 & 12.22 & 664 & 8.58 & 11.43 & 11.18 & 548 \\
\hline$\ldots$ & $\ldots$ & $\ldots$ & $\ldots$ & $\ldots$ & $\ldots$ & $\ldots$ & $\ldots$ & $\ldots$ & $\ldots$ & $\ldots$ & $\ldots$ & $\ldots$ \\
\hline
\end{tabular}

(a) Product characteristics

\begin{tabular}{ccccccc}
\hline \multicolumn{2}{c}{ Machine 1 } & \multicolumn{2}{c}{ Machine 2 } & \multicolumn{2}{c}{ Machine 3 } \\
\hline $\begin{array}{l}\text { Flexible transfer } \\
\text { line configurations }\end{array}$ & MTTR $_{1}$ & MTTF $_{1}$ & MTTR $_{2}$ & MTTF $_{2}$ & MTTR $_{3}$ & MTTF $_{3}$ \\
\hline 1 & 64.09 & 186.54 & 32.03 & 95.69 & 89.44 & 266.33 \\
2 & 44.38 & 110.97 & 77.95 & 202.61 & 97.56 & 243.64 \\
3 & 75.84 & 184.93 & 91.28 & 215.53 & 64.03 & 151.36 \\
4 & 37.66 & 98.61 & 55.52 & 132.64 & 97.40 & 230.21 \\
5 & 70.20 & 210.16 & 73.30 & 171.24 & 57.22 & 160.67 \\
6 & 41.43 & 111.10 & 44.82 & 123.31 & 89.67 & 242.02 \\
7 & 97.66 & 260.78 & 73.81 & 188.56 & 81.71 & 197.01 \\
8 & 91.17 & 253.90 & 76.53 & 218.88 & 61.67 & 153.67 \\
9 & 35.16 & 94.86 & 96.44 & 231.44 & 91.47 & 226.89 \\
10 & 43.88 & 117.98 & 44.94 & 130.13 & 33.32 & 83.75 \\
$\ldots$ & $\ldots$ & $\ldots$ & $\ldots$ & $\ldots$ & $\ldots$ & $\ldots$ \\
\hline
\end{tabular}

(b) Machine characteristics $\left(\mathrm{UTR}_{\mathrm{i}} \in[70,75 \%]\right)$

Table 1. Randomly generated configurations for a 3-product, 3-machine flexible transfer line. 


\begin{tabular}{ccccccccccccc}
\hline & \multicolumn{4}{c}{ UTR $(\%)$} & & \multicolumn{2}{c}{ UTR_RE (\%) } & \multicolumn{3}{c}{$\begin{array}{c}\text { Accepted Student's } \\
\text { t-tests }\end{array}$} \\
\hline $\begin{array}{c}\text { Flexible transfer } \\
\text { line configuration }\end{array}$ & $\begin{array}{c}\text { Simula- } \\
\text { tion }\end{array}$ & DGL & GDLB & C\&Y & DGL & GDLB & C\&Y & DGL & GDLB & C\&Y \\
\hline 1 & 51.3257 & 51.3317 & 44.3094 & 49.6500 & 0.0116 & 13.67 & 3.264 & 1 & 0 & 0 \\
2 & 48.7596 & 48.7965 & 32.6310 & 45.7645 & 0.0756 & 33.07 & 6.142 & 1 & 0 & 0 \\
3 & 46.1971 & 46.1353 & 34.8636 & 44.3138 & 0.1339 & 24.53 & 4.076 & 1 & 0 & 0 \\
4 & 47.9910 & 48.0207 & 35.3360 & 44.9718 & 0.0617 & 26.36 & 6.291 & 1 & 0 & 0 \\
5 & 49.9129 & 49.8166 & 36.8001 & 47.2092 & 0.1928 & 26.27 & 5.416 & 1 & 0 & 0 \\
6 & 49.3297 & 49.3996 & 41.3548 & 47.4634 & 0.1416 & 16.16 & 3.783 & 1 & 0 & 0 \\
7 & 49.1540 & 49.1015 & 35.6781 & 45.8573 & 0.1067 & 27.41 & 6.706 & 1 & 0 & 0 \\
8 & 50.2187 & 50.1821 & 32.8583 & 47.3924 & 0.0729 & 34.56 & 5.627 & 1 & 0 & 0 \\
9 & 48.2657 & 48.3657 & 38.2332 & 45.6510 & 0.2071 & 20.78 & 5.417 & 1 & 0 & 0 \\
10 & 50.5743 & 50.6281 & 35.1972 & 47.2779 & 0.1063 & 30.40 & 6.517 & 1 & 0 & 0 \\
$\ldots$ & $\ldots$ & $\ldots$ & $\ldots$ & $\ldots$ & $\ldots$ & $\ldots$ & $\ldots$ & $\ldots$ & $\ldots$ & $\ldots$ \\
\hline
\end{tabular}

GDLB: refers to the equivalent technique by Gershwin [18], Dallery et al. [19], and Liu and Buzacott [20] C\&Y: refers to the proposal by Chen and Yuan [21], DGL: refers to the proposed technique.

Table 2. Comparison of simulation results with analytical results for production line configurations of Table 1 


\begin{tabular}{|c|c|c|c|c|c|c|c|c|}
\hline & & \multirow{2}{*}{$\begin{array}{l}\text { Number } \\
\text { of } \\
\text { Machines }\end{array}$} & \multicolumn{2}{|c|}{ DGL Technique } & \multicolumn{2}{|c|}{ GDLB Technique } & \multicolumn{2}{|c|}{ C\&Y Technique } \\
\hline & & & $\begin{array}{c}\text { UTR_MRE } \\
(\%)\end{array}$ & $\begin{array}{c}\text { Accepted } \\
\text { Student's } \\
\text { t-tests } \\
(\%)\end{array}$ & $\begin{array}{c}\text { UTR_MRE } \\
(\%)\end{array}$ & $\begin{array}{c}\text { Accepted } \\
\text { Student's } \\
\text { t-tests } \\
(\%)\end{array}$ & $\begin{array}{c}\text { UTR_MRE } \\
(\%)\end{array}$ & $\begin{array}{c}\text { Accepted } \\
\text { Student's } \\
\text { t-tests } \\
(\%)\end{array}$ \\
\hline \multirow{24}{*}{ 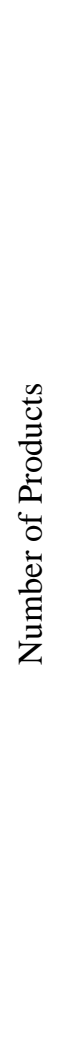 } & \multirow{8}{*}{3} & 3 & 0.08821065 & 100 & 24.0221588 & 0 & 5.42910316 & 0 \\
\hline & & 4 & 0.08816368 & 100 & 29.5900747 & 0 & 6.83983041 & 0 \\
\hline & & 5 & 0.09133787 & 100 & 32.1601839 & 0 & 7.80488687 & 0 \\
\hline & & 6 & 0.10799181 & 100 & 35.1050614 & 0 & 8.66105825 & 0 \\
\hline & & 7 & 0.12062543 & 100 & 37.5830161 & 0 & 9.27135782 & 0 \\
\hline & & 8 & 0.13620527 & 100 & 38.5700564 & 0 & 9.78397356 & 0 \\
\hline & & 9 & 0.13917206 & 100 & 40.7055591 & 0 & 10.2303786 & 0 \\
\hline & & 10 & 0.14976247 & 100 & 41.2736451 & 0 & 10.6079263 & 0 \\
\hline & \multirow{8}{*}{5} & 3 & 0.05990899 & 100 & 23.5021912 & 0 & 5.24570065 & 0 \\
\hline & & 4 & 0.06637277 & 100 & 29.5775932 & 0 & 6.84445426 & 0 \\
\hline & & 5 & 0.07891914 & 100 & 32.3083462 & 0 & 7.76514574 & 0 \\
\hline & & 6 & 0.09411104 & 100 & 34.9198968 & 0 & 8.70077252 & 0 \\
\hline & & 7 & 0.11360067 & 100 & 37.5619280 & 0 & 9.23199331 & 0 \\
\hline & & 8 & 0.09989136 & 100 & 38.8445349 & 0 & 9.91370695 & 0 \\
\hline & & 9 & 0.12506294 & 100 & 40.6375025 & 0 & 10.2996609 & 0 \\
\hline & & 10 & 0.16114315 & 100 & 41.4739280 & 0 & 10.7721840 & 0 \\
\hline & \multirow{8}{*}{9} & 3 & 0.05284090 & 100 & 23.7800632 & 0 & 5.33554311 & 0 \\
\hline & & 4 & 0.06757900 & 100 & 29.0729848 & 0 & 6.85011550 & 0 \\
\hline & & 5 & 0.07106429 & 100 & 32.4279961 & 0 & 7.86560995 & 0 \\
\hline & & 6 & 0.08691862 & 100 & 35.3835930 & 0 & 8.73469392 & 0 \\
\hline & & 7 & 0.07783048 & 100 & 37.4666852 & 0 & 9.33743741 & 0 \\
\hline & & 8 & 0.07744580 & 100 & 39.1037550 & 0 & 9.96193380 & 0 \\
\hline & & 9 & 0.10605383 & 100 & 40.6294297 & 0 & 10.3656059 & 0 \\
\hline & & 10 & 0.10585076 & 100 & 41.4971570 & 0 & 10.6632000 & 0 \\
\hline
\end{tabular}

Table 3. UTR mean relative errors and accepted Student's t-tests resulting from comparing simulation results with studied analytical techniques $\left(\mathrm{UTR}_{\mathrm{i}} \in[70,75 \%]\right)$

\begin{tabular}{|c|c|c|c|c|c|c|c|c|}
\hline & & \multirow{2}{*}{$\begin{array}{l}\text { Number } \\
\text { of } \\
\text { Machines }\end{array}$} & \multicolumn{2}{|c|}{ DGL Technique } & \multicolumn{2}{|c|}{ GDLB Technique } & \multicolumn{2}{|c|}{ C\&Y Technique } \\
\hline & & & $\begin{array}{c}\text { UTR_MRE } \\
(\%)\end{array}$ & $\begin{array}{c}\text { Accepted } \\
\text { Student's } \\
\text { t-tests } \\
(\%)\end{array}$ & $\begin{array}{c}\text { UTR_MRE } \\
(\%)\end{array}$ & $\begin{array}{c}\text { Accepted } \\
\text { Student's } \\
\text { t-tests } \\
(\%)\end{array}$ & $\begin{array}{c}\text { UTR_MRE } \\
(\%)\end{array}$ & $\begin{array}{c}\text { Accepted } \\
\text { Student's } \\
\text { t-tests } \\
(\%)\end{array}$ \\
\hline \multirow{6}{*}{ 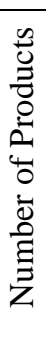 } & & 3 & 0.0469301 & 100 & 26.817553 & 0 & 0.70604954 & 0 \\
\hline & 3 & 10 & 0.0912515 & 100 & 60.508538 & 0 & 2.74390757 & 0 \\
\hline & 5 & 3 & 0.0610456 & 100 & 26.790125 & 0 & 0.74570065 & 0 \\
\hline & 5 & 10 & 0.0809626 & 100 & 60.830587 & 0 & 2.77218403 & 0 \\
\hline & 0 & 3 & 0.0763310 & 100 & 26.563491 & 0 & 0.71180387 & 0 \\
\hline & 9 & 10 & 0.0720817 & 100 & 60.717858 & 0 & 2.68683933 & 0 \\
\hline
\end{tabular}

Table 4. UTR mean relative errors and accepted Student's t-tests resulting from Comparing simulation results with studied analytical techniques $\left(\mathrm{UTR}_{\mathrm{i}} \in[95,100 \%]\right)$ 